\title{
New technologies challenge the future of taxonomy in Orthoptera*
}

Submitted April 4, accepted June 7, 2010

\author{
M.M. CIGLIANO AND D. EADES
}

(MMC) División Entomología, Museo de La Plata, Paseo del Bosque S/N, 1900, La Plata, Argentina.

E-mail: cigliano@fcnym.unlp.edu.ar

(DE) 1816 South Oak Street, Champaign, IL 61820.

* Plenary Lecture presented 10th International Congress of

Orthopterology. Antalya, Turkey, 21-25 June 2009.

\begin{abstract}
A global imperative for the conservation of biodiversity brings into focus the need for taxonomic research. However, this biodiversity crisis is reflected in a parallel taxonomic crisis. Whereas molecular information is increasingly the evidential basis for delimiting species, revisionary taxonomy is frequently dismissed as merely 'descriptive' and lacking a hypothesis-driven nature. Phylogenetic classifications are optimal for storing and predicting information, but phylogeny divorced from taxonomy is unrealizable. Taxonomy, systematics, and phylogeny are interwoven, hypothesis-driven sciences with a shared theoretical base. Taxonomic knowledge remains essential to biological research and knowledge acquisition is made urgent by the biodiversity crisis. Taxonomy needs to prepare to take advantage of new information technology capabilities. Rapid advances in bioinformatics have provided unprecedented opportunities to conduct taxonomic research more efficiently; cybertaxonomy is emerging as an exciting new branch. Here we argue the great potential for using the Orthoptera Species File online (http://orthoptera.speciesfile.org/) as a tool for monograph and revisionary studies of Orthoptera and we also draw attention to a method of integrating many cybertaxonomic tools with species descriptions: this to engage both the specialist taxonomic community and a wider public in the gathering and deepening understanding of taxonomic knowledge.
\end{abstract}

\section{Key words}

biodiversity, taxonomy, bioinformatics, cybertaxonomy, species file

\section{Introduction}

Society has a growing need for taxonomic information in order to conserve, manage, understand and enjoy the natural world. At the same time, support for taxonomy and museum collections is failing to keep pace (Wheeler et al. 2004). Understanding the origin and diversity of the living world is an endeavor that requires a revision of traditional taxonomic practices. However, it is widely recognized that progress in the study of biodiversity in this time of crisis is hindered by a worldwide shortage of taxonomists, coupled with a lack of sufficient funding for taxonomic study (the "Taxonomic impediment" Lipscomb et al. 2003, Wheeler 2004, Carvalho et al. 2005, Crisci 2006). To overcome the impediment, it is mandatory both to adopt new technologies developed in other fields for taxonomic use, and to address the key conceptual challenges faced by taxonomy.

During the second half of the $20^{\text {th }}$ century, taxonomy reinvented itself as a modern science (Godfray et al. 2007). This reinvention had two main components: first was the proposal (now almost universally accepted) that classification should reflect phylogeny. Evolution had been at the heart of taxonomy since Darwin, but Willi Hennig made the connection more formal. The phylogenetic systematics pioneered by Hennig in the 1950s and 1960s provided a conceptual framework and a set of tools enabling organisms to be classified in a consistent manner, one that explicitly took account of evolutionary relationships. Critically, every taxonomic decision, from a species definition to a system of higher classification, was to be treated as a provisional hypothesis, potentially falsifiable by new data. Taxonomy was brought into the fold of 'hypothesis-driven science' (Godfray \& Knapp 2004).

The second component of taxonomy's reinvention was the molecular revolution. Molecular data provide a new category of features that allow not only reconstruction of closely related taxa, but also broad-scale comparisons. Unquestionably, we are witnessing an extraordinary time as molecular systematics makes an unprecedented revolution within the discipline. In fact, systematics has transformed biology through its contribution of a phylogenetic perspective. For taxonomists working on living organisms there is no question but that molecular techniques are now central to understanding evolutionary relationships.

However, taxonomy is not only phylogeny (Crisci 2006): it also involves the definition and description of species, and the organization of knowledge in a manner that can be used by the wider community. And though practically useful, descriptive taxonomy is not just a service agency for the rest of biology (Wilson 2004). Its output is far more than a stock inventory of Earth's biodiversity. Among its cascade of derivative functions, taxonomy lays the foundations for the phylogenetic tree of life; it provides a requisite database for ecology and conservation science; it makes accessible the vast, and still largely unused benefits, offered by biodiversity to humanity. Nowadays, there is a rejoining of new technological advances with urgent needs, making this an ideal time to revitalize comparative morphology and bring about a renaissance in taxonomy. Rapid advances in cyber-infrastructure, bioinformatics, digital imaging and related information technologies, mean that taxonomists and the museum community can set ambitious goals for their research, making accessible existing information and generating new information on a scale appropriate to address the problem of declining biodiversity. However, technology and web initiatives are necessary and useful - so long as they aim to enhance the taxonomic research, not aspire to replace it.

In recent years there has been a significant increase of interest in the field of biodiversity informatics (Smith et al. 2009) and several informatics initiatives have been developed [Antweb; AmphibiaWeb; Avibase; Barcode of Life Initiative; Biodiversity Heritage Library; Fishbase; Global Biodiversity Information Facility (GBIF); Biodiversity Information Standards (TDWG); Encyclopedia of 
Life (EOL); European Distributed Institute of Taxonomy (EDIT); GenBank; Species 2000, ITIS Catalogue of Life (CoL); MorphBank; MorphoBank; Scratchpads; TreeBase; ZooBank].

Some of these are large data aggregations, while others address special-purpose data. However, only a very few of these projects actually integrate the diverse kinds of data that are produced and can be used by systematists of specific taxonomic groups. One such exception is the Orthoptera Species File online (OSF), managed and developed by David Eades.

The OSF was originally founded by Daniel Otte, who published the first volume of the Orthoptera Species File in 1994 to cover crickets (Grylloidea) (Otte 1994). Subsequent volumes continued through volume eight in 2000, completing coverage of all Orthoptera (Otte, 2000). The collaboration between Otte and Piotr Naskrecki led to 'Version One' of the Orthoptera Species File Online, first posted to the Internet on October 20, 1997. David Eades was responsible for a new design and new versions of OSF: these provide cross-checking during the addition of new data, in a way that identifies and halts nomenclatural errors; his design also greatly reduces the number of changes that must be entered manually (Eades \& Otte 2010).

The Orthoptera Species File online provides a space on the web that brings together taxonomic information on Orthoptera, without the limitations of traditional paper-based publications. From its inception the strength and value of OSF to the taxonomic community has been its integration of synonyms and taxonomic literature, for all taxa within it, with digital images of type specimens, interactive keys, sound recordings, and maps. The Orthoptera Species File is a website and a database created specifically to provide a source of taxonomic information for researchers and others working on Orthoptera, and to provide software useful for other taxonomic groups. The underlying structure of the website and database is Species File Software (http://software.speciesfile.org). The present paper refers just to Orthoptera, but the features described are available for any and all groups for which species files have been developed. This includes aphids, leaf-legged bugs, and all Polyneoptera except termites. Other species files are in early stages of development.

Recently, a feature of OSF (developed by David Eades and the group at the Illinois Natural History Survey, University of Illinois) added an extremely valuable taxonomic tool that supplements existing resources for conducting systematic studies within Orthoptera. This new feature enables an author to add data temporarily in a "private" Species File Online, while conducting a systematic study of any group/taxa of Orthoptera. This information remains hidden from the public until its publication. This new development will also enhance access to taxonomically relevant information through the incorporation in the publication of embedded hyperlinks to different parts of the Orthoptera Species file.

Herein we describe this new vision for data publication and dissemination in orthopteran taxonomy. In order to develop and apply innovative ways of publishing, we describe the process applied for the first time in Cigliano et al. (2010) and Cigliano \& Amedegnato (in press). The central core is that all primary biodiversity data underlying a taxonomic paper can be added to OSF, and the paper can then be edited, including embedded links to this information displayed in OSF.

\section{Implementation}

1. At an early stage in preparation of a manuscript (prior to its publication) a "private" Species File is generated at the server (Species File Software server at the Illinois Natural History Survey, USA). This file includes all the relevant information from OSF for the selected taxon.

2. A second step relates to the editing of nomenclatural and basic taxon data. The author of the systematic revision adds/edits the data and information relevant to the new species/taxa described in the manuscript, into the "private" Species File online. The new taxa added need to be congruent with the currently recognized higher classification in OSF.

3. The third step involves additions to the 'private' Species File online of information that illustrates the taxa:

3.a. Of illustrations - photographs or drawings - of type specimens, as well as other images of diagnostic characters.

3.b. Of georeferenced specimen data. An occurrence dataset is added. Recently, a new feature has been implemented in OSF that allows the visualization of specimen-based distribution maps using the technology of Google maps.

3.c. Of interactive keys, including color images and drawings illustrating the diagnostic characters.

4. In step 4 editing of the manuscript takes place, including embedded links to images of type specimens, maps based on geo-referenced specimen data, and interactive keys contained in the "private" Species File online.

5. The manuscript is submitted for peer-review to a journal that has an online version in addition to its printed version and allows the inclusion of embedded hyperlinks. At this time, the information of the new taxa can only be seen at the "private" Species File online by the editor and reviewers of the journal to which the paper is submitted. Editors and reviewers are given special confidential registrations that allow access for viewing the data, but do not allow editing.

6. Once the manuscript is accepted for publication, the embedded hyperlinks of the manuscript have to be changed for the definitive hyperlinks that will point at different parts of OSF. The following steps/programs are part of this procedure:

6.a. Reserve IDs for a future merge into OSF: this program is executed when the work on the "private" Species File is completed and the manuscript is ready for final revision. This program reserves appropriate IDs in the public website (OSF). After this is accomplished, it is possible to enter the "private" Species File and see the association between existing IDs in the private species file and the reserved IDs in the public website that will be used after the merge.

6.b. Show IDs reserved in OSF for when the "private" Species File is merged into it. In this way the embedded hyperlinks that are pointing at different parts of the "private" Species File can be changed in the manuscript to the definitive hyperlinks that will point at the corresponding parts in OSF.

7. The final version of the manuscript with the definitive hyperlinks pointing at different parts of OSF is submitted to the journal for typesetting the proofs of the manuscript and definitive publication.

8. Simultaneously to the publication of the paper, the "private" Species File is merged into OSF. After this program is run the embedded hyperlinks in the paper published become active and point to the different parts of the OSF public website.

Assistance in editing of data in OSF can be provided by the group responsible for data management at the "Museo de La Plata", Argentina. 


\section{Concluding remarks}

The urgency of taxonomic research, on not just Orthoptera but all organisms, arises from the environmental ravages of the biodiversity crisis. Taxonomy is of major importance for progress in conservation and biological research generally (Samways 1997, 2005). However, description of new species and new taxa requires considerable investment of time, energy, knowledge and resources. Similarly, the collection of specimen records requires serious efforts. Each species-by-occurrence record collected on Earth has its own value and deserves proper registration, publication, preservation and dissemination (Penev et al. 2009).

We believe our proposed approach will motivate authors to include these new features in their publication of new orthoptera taxa. This will enhance the information content of the publication in the following ways:

-by creating links to images of morphological diagnostic characters, supplementing those included in the publication displayed in OSF.

-by creating links to sound recordings displayed in OSF.

-by creating links to high-resolution color images of type specimens of species described in the manuscript; these images now can be added only in papers published in journals avoiding the cost of color plates.

-by creating links to interactive identification keys illustrated with color images.

-by creating links to maps displayed with Google map technology, based on geo-referenced specimen data.

-by allowing co-authors that collaborate on the same projects to simultaneously add information to a "private" Species File, while working at different museums or institutions anywhere in the world.

-by ensuring that the products of any systematic research will be comprised of the publication plus the digital taxon "knowledge bases", so including effectively the sum total of knowledge and information about the group studied.

During the last two and a half centuries, Linneaus' (1758) system for naming, ranking and classifying organisms has allowed us to document the pattern of life on Earth. Darwin's (1859) theory of evolution has helped us to understand the process of how this diversity of life came about, and Hennig's (1966) phylogenetic methodology has enabled us to reconstruct the tree of life.

During the last two decades or so, the internet has dramatically changed the way we work. The rapid advances in bioinformatics have provided taxonomists unprecedented opportunities to do our research more efficiently and make our findings more accessible. Cybertaxonomy is emerging as an exciting new branch of taxonomy. OSF represents a useful tool to make our systematic work more visible. Through this registry of properties of Orthoptera species, the community gains open access to the world's total knowledge of species, and can share the wonders of the biological diversity of Orthoptera.

As taxonomy evolves into the 'cyber' age, it is reappearing in the public eye. The future of systematics will involve advances in computational photography, innovations in creating identification keys, expert-knowledge systems and, eventually, genetic identification gadgets. Soon, the practice of taxonomy will be unthinkable without the Internet.

However, even though new bioinformatics standards can bring new tools to systematists, taxonomic knowledge remains essential to biological research (Raven 2004). We have to ensure that regional species inventories of Orthoptera are undertaken in an ambitious and coordinated manner. Varied approaches can complement one another, achieve particular aims and appeal to different funding sources. Such inventories must be guided by existing taxonomic knowledge, so that we prioritize field work to fill gaps in our inventory of Orthoptera species. New tools can be incorporated to allow the taxonomic work to be more efficient and supplement the existing taxonomic resources. But no matter how good these new tools are, what is at the very foundation cannot be replaced: systematic revisions and monographs should remain central to taxonomy. Poor systematic hypotheses, after all, will lead to erroneous conservation priorities and spurious ecological and biological studies.

\section{Acknowledgements}

We thank Holger Braun, Cristina Damborenea and Marta Fernández for reading this manuscript and providing feedback.

\section{References}

AmphibiaWeb. http://amphibiaweb.org/

Avibase-the world database. http://avibase.bsc-eoc.org/

Barcode of Life Initiative. http://www.dnabarcodes.org/

Biodiversity Heritage Library http://www.biodiversitylibrary.org/

Carvalho M.R., De Bockmann F.A., Amorin D.S., De Vivo M., De Toledo Piza M., Menezes N.A., De Figueiredo R.M., Castro C., Gill A.C., Mceachran J.D., Compagno L.J.V., Schell R.C., Britz R., Lundberg J.G., Bari R.P., Nelson G. 2005. Revisiting the taxonomic impediment. Science 307: 353.Catalogue of Life: Annual Checklist. Species 2000. ITIS. http: //www.catalogueoflife.org/

Cigliano M.M., Amedegnato C., Pocco M.E., Lange C.E. 2010. Revisionary study of Pediella Roberts (Orthoptera: Acrididae: Melanoplinae) from the Andes Highlands. Zootaxa 2431: 51-61.

Cigliano M.M., Amedegnato C. The high Andean Jivarus Giglio Tos (Orthoptera, Acridoidea, Melanoplinae): Systematics, phylogenetic and biogeographic considerations. Systematic Entomology. (in press)

Crisci J.V. 2006. One-dimensional systematist: perils in a time of steady progress. Systematic Biology 31: 217-221.

Darwin C. 1859. The Origin of Species by Means of Natural Selection. John Murray, London.

Eades D.C., Otte D. Orthoptera Species File Online. Version 2.0/3.5. 2010. $<$ http://Orthoptera.SpeciesFile.org>

Encyclopedia of Life (EOL). http://eol.org/

European Distributed Institute of Taxonomy (EDIT). http://www.etaxonomy.eu/

FishBase. http://www.fishbase.org/

GenBank. http://www.ncbi.nlm.nih.gov/Genbank/

Godfray H.C.J. 2002. Challenges for taxonomy. Nature 417: 17-19.

Gotelli N.J. 2004. A taxonomic wish-list for communityecology. Philosophical Transactions of the Royal Society of London B. 359: 585-597.

Godfray H.C.J., Knapp S. 2004. Taxonomy for the twenty-first centuryIntroduction. Philosophical Transactions Royal Society of London. B 359: 559-569.

Global Biodiversity Information Facility (GBIF). http://www.gbif.org/

Hennig W. 1966. Phylogenetic Systematics. Univ. Illinois Press, Urbana.

Lipscomb D., Platnick N., Wheeler Q. 2003. The intellectual content of taxonomy: a comment on DNA taxonomy. Trends in Ecology and Evolution 18: 65-66

Linnaeus C. 1758. Systema Naturae. 100 Edition, Stockholm.

Mace G.M. 2004. The role of taxonomy in species conservation. Philosophical Transactions Royal Society of London B. 359: 711-719.

Miller J.A., Griswold C.E., Yin C.M. 2009. The symphytognathoid spiders of the Gaoligongshan, Yunnan, China (Araneae, Araneoidea): systematics and diversity of micro-orbwearvers. ZooKeys 11: 9-195.

MorphBank. http://www.morphbank.net/

MorphoBank. http://morphobank.org/ 
Otte D. 1994. Orthoptera Species File. Number 1. Crickets (Grylloidea). Publications on Orthopteran Diversity, 120 pp.

Otte D. 2000. Orthoptera Species File. Number 8. Grillacrididae, Stenopelmatidae, Cooloolidae, Schizodactylidae, Annastostomatidae and Rhaphidophoridae. Publications on Orthopteran Diversity, 97 pp.

Penev L., Erwin T., Miller J., Chavan V., MoritzT., Griswald C. 2009. Publication and dissemination of datasets in taxonomy: ZooKeys working example. ZooKeys 11: 1-8.

Pyle R.L., Earle J.L., Green BD. 2008. Five new species of the damselfish genus Chromis (Perciformes: Labroidei: Pomacentridae) from deep coral reefs in the tropical western Pacific. Zootaxa: 1671: 3-31

Raven P. 2004. Taxonomy: where are we now? Philosophical Transactions Royal Society of London. B 359: 729-730.

Scratchpads. Biodiversity online. http://scratchpads.eu/

Samways M. 1997. Conservation biology of Orthoptera, pp. 481-496. In Gangwere S. K., Muralirangan M.C., Muralirangan M. (Eds). The Bionomics of Grasshoppers, Katydids and their Kin. Wallingford: CAB International.

Samways M. 2005. Insect Diversity Conservation. Cambridge University Press.

Smith V.S., Rycroft S.D., Harman K.T., Scott B., Roberts D. 2009. Scratchpads: a data-publishing framework to build, share and manage information on the diversity of life. BMC Bioinformatics 2009, 10 (Suppl 14): S6.

Taxonomic Database Working Group (TDWG). http://www.tdwg.org/

TreeBase. A database of Phylogenetic knowledge. http:// www.treebase.org/

Wheeler Q.D. 2004. Taxonomic triage and the poverty of phylogeny. Philosophical Transactions Royal Society of London B 359: 571-583.

Wheeler Q.D., Raven P.H., Wilson E.O. 2004. Taxonomy: impediment or expedient? Science 303: 285.

Wilson E.O. 2004. Taxonomy as a fundamental discipline. Philosophical Transactions Royal Society of London B 359: 739.

ZooBank. http://zoobank.org/ 